\title{
Human retroviruses in the second decade: A personal perspective
}

\author{
Human retroviruses have developed novel strategies for their propagation and survival. \\ A consequence of their success has been the induction of an extraordinarily diverse set \\ of human diseases, including AIDS, cancers and neurological and inflammatory \\ disorders. Early research focused on their characterization, linkage to these diseases, \\ and the mechanisms involved. Research should now aim at the eradication of human \\ retroviruses and on treatment of infected people.
}

Retroviruses are transmitted either genetically (endogenous form) or as infectious agents (exogenous form) ${ }^{1,2}$. As do many other animal species, humans have both forms $s^{3,4}$. In general, endogenous retroviruses are evolutionary relics of old infections and are not known to cause disease. The DNA of many species, including humans, harbours multiple copies of different retroviral proviruses. The human endogenous proviral sequences are virtually all defective, and comprise about one percent of the human genome, though R. Kurth's group in Frankfurt showed that one, called HERV-K, can code for all typical retroviral gene products and form particles ${ }^{4}$. Future interest in these sequences will depend on demonstrating some function or role in disease.

The infectious retroviruses are, of course, another story. In humans they may directly cause leukaemias, neurological diseases, inflammatory disorders and immune deficiency, as well as enhancing the incidence of other cancers (including B-cell lymphoma, cancer of the liver, and cervical cancer, indirectly, presumably by fostering replication of other viruses. There are two groups of infectious human retroviruses: the human T-cell leukaemia/lymphotropic viruses (HTLV-I and HTLV-II) belonging to the oncoretroviridae and the human immunodeficiency viruses (HIV-1 and HIV-2) belonging to the lentiretroviridae ${ }^{5-12}$. In this review, HTLV refers to HTLV-1; likewise, HIV refers to HIV-1.

\section{Similarities and contrasts}

As do all retroviruses, human retroviruses replicate through a DNA intermediate (the provirus), which integrates in the target cell DNA where it may remain transcriptionally silent. In fact, latency may be the chief means by which the HTLVs escape immune detection. HIV infections are, however, often not latent. Their survival also involves chronic high-level replication and steady destruction of the immune system. The routes of transmission of HTLV-I, the first discovered human retrovirus ${ }^{5,12}$, were shown to be through sex, from mother to child and via contaminated blood, similar to the routes later shown for HIV1. HTLV-I is endemic in many parts of the world, including equatorial Africa, southern Japan, Central and South America, and in the immigrant descendants of people from these regions. Recent molecular epidemiological studies revealed several subtypes, a prevalent type called 'cosmopolitan' HTLV-I and other subtypes endemic in parts of the South Pacific and equatorial Africa $^{13}$. Although distinguishing these isolated subtypes of HTLV-I may be only of academic interest, it remains possible that they may cause unexpected diseases. A year after the discovery of HTLV-I, a second human retrovirus, HTLV-II, was discovered ${ }^{14}$. Though its characteristics are strikingly similar to HTLV-I, HTLV-II is not so clearly linked to human disease. It is curious that HTLV-II is endemic in some American Indians and more prevalent in drug addicts than HTLV- $\mathrm{I}^{15.16}$.

HIV-1 is also most prevalent in equatorial Africa, but in contrast to HTLV the demography of the HIV epidemic is still in flux, and the virus is new to most of the world. The number of infected people worldwide is now estimated to be about 17 million and is predicted to reach 30 to 50 million by the year 2000 . However, precise predictions are not possible because of varying human behaviour and cofactors for transmission. The far greater variability of HIV as compared with HTLV also leaves open the possibility of emergence of variants with greater sexual transmissibility, as suggested by Max Essex (pers. commun.).

Both the HTLVs and HIVs have counterparts in Old World monkeys known as simian T-cell leukaemia virus type I (STLVI), and simian immunodeficiency virus (SIV), respectively. As a result, studies of human retroviruses in these animals have been of interest ${ }^{13}$.

In vitro as well as in vivo the most dramatic effects of both HTLVs and HIVs are seen on the CD4+ T cell. Although HTLV infects several T-cell subtypes (its receptor remains unknown), it chiefly immortalizes the $\mathrm{CD} 4^{+} \mathrm{T}$ cell which is the same cell type to develop leukaemia. The major receptor for HIV is CD4. Consequently, HIVs infect chiefly the CD4+ T cell and cells of the monocyte/macrophage lineage that also express CD4. Other cell types may be infected, perhaps mediated by Fc or complement receptors in the presence of antiviral antibodies. In contrast to HTLV-I, HIV is cytopathic for CD4+ T cells often inducing massive syncytia formation and abruptly killing the infected cell after the cell is activated ${ }^{17}$. A difference between the two groups of human retroviruses is the relative ease of transmission by HIV as cell-free virions compared with the HTLVs, which are probably only transmitted by infected cells. This difference may account for the more rapid spread of HIV.

Both HTLVs and HIVs take years from the time of infection to clinical disease, but for HIV there is no real latency. From in situ studies in 1985 we have known some infected cells are always expressing virus, and more recently we have learned that there is much more ongoing HIV-1 replication and cell killing than previously thought to occur ${ }^{18,19}$, especially in lymph nodes $^{20,21}$.

\section{HTLV as a cause of leukaemia}

HTLV-I was established as the cause of an aggressive adult T-cell leukaemia (ATL) in 1980-81 (refs 10-12, 22, 23), just before 
detection of the first cases of AIDS. About two to five per cent of infected people per 70-year life span develop leukaemia. A similar number may develop serious neurological or inflammatory disease. It has recently been suggested that leukaemia develops in individuals infected in infancy by maternal transmission, in contrast to the neurological disorders, which are more apt to occur following adult infection by sexual transmission $^{24}$. Because HTLVs immortalize some infected $\mathrm{T}$ cells and because the Tax protein transactivates not only HTLV-I genes but several cellular genes, including interleukin-2 (IL-2) and the alpha chain of the IL- 2 receptor (IL-2R $\alpha$ ), Tax has been invoked as the key to the events leading to T-cell immortalization ${ }^{25,26}$. However, because IL-2 production is only transient, other events must be involved in the eventual IL- 2 independent Tcell proliferation. A candidate mechanism involves a newly discovered HTLV-I protein, p12, which binds to the beta chain of the IL-2R in the cytoplasmic domain of a region connected to a signal transduction pathway ${ }^{13}$. This and other newly found HTLV proteins are products of messenger RNA (mRNA) formed by using different splice sites from the major mRNA. Tax also transactivates genes for many other cytokines and HLA. Although it is not easy to speculate on the overall effects of the activation of a large number of cytokines by Tax, one, IL-7, also promotes T-cell proliferation utilizing the gamma chain of the IL-2R. Other effects of Tax may lead to genetic diversity of infected cells, and some of these changes may promote greater cell proliferation and consequently a greater chance for neoplastic transformation ${ }^{27}$. Slowly, the events leading to T-cell immortalization are being elucidated, but immortalization or even transformation of a cell is not equivalent to development of leukaemia, which undoubtedly requires further genetic changes. In the absence of evident cofactors or genetic predispositions, we can only speculate why some develop adult T-cell leukaemia and others do not. The failure to generate many virions coupled with its poor infectiousness presents the need for HTLV to expand and perhaps diversify the infected T-cell population for its survival. The sum effects of these activities (increased cell proliferation and genetic instability) increase the risk of neoplastic conversion. However, this should indeed be a rare and chance occurrence, as it has to be balanced against Tax activation of HTLV production and consequent immune detection and destruction of the cell.

\section{HTLV-I and neurological disease}

A few neurological diseases are associated with HTLV-I infection. The most common and severe is tropical spastic paraparesis (TSP) also referred to as HTLV-I-associated myelopathy (HAM), a demyelinating disorder resembling multiple sclerosis (MS) ${ }^{28,22}$. Virus is generally not found in cells of the central nervous system (CNS), except for some infiltrating $T$ cells. People with TSP usually have more HTLV-I replication and correspondingly a greater immune response to the virus compared with infected asymptomatic people and ATL patients. This finding suggests a possible autoimmune mechanism, which was supported by the finding of $\mathrm{CD}^{+}$cytotoxic T lymphocytes (CTLs) with specificity for Tax or Env in the cerebrospinal fluid. Although there is no clear evidence for a retroviral cause of MS, knowing the pathogenesis of TSP/HAM may provide clues to its pathogenesis.

Other HTLV-I-associated diseases include infective dermatitis of children, lung inflammation, uveitis, rheumatoid arthritis and polymyositis. Whether these inflammatory disorders de- velop as a result of Tax activation of cytokines in T cells infiltrating specific tissue sites, autoimmune phenomena, other disturbances of the immune system or some other mechanism is unknown and, as for MS, the ultimate importance of HTLV in these illnesses may be in elucidating molecular mechanisms for their more common (non-HTLV associated) forms.

\section{Therapy and prevention of HTLV-I disease}

Adult T-cell leukaemia is not curable despite extensive information on its cause and pathogenesis and on the properties of the leukaemic cell. Most therapy is based on combination cytotoxic chemotherapy, although Waldmann and colleagues have pioneered a rational approach to delivering cell-killing radionucleotides selectively to the ATL cell by targeting their constitutively expressed IL-2R $\alpha$ chain $^{30}$. Others have used a combination of azidothymidine (AZT) and interferon alpha (IFN- $\alpha$ ), presumably as antiviral agents ${ }^{31,32}$. Remission of the leukaemia occurred in most patients. However, after development of ATL, HTLV-I replication is not needed to maintain the leukaemia. Although the logic of this approach might be faulted, the results so far cannot be. AZT and IFN- $\alpha$ not only interfere with virus replication, they also affect cell metabolism. It will be of importance to elucidate their synergistic effects on ATL cells.

The legacy of HTLV-I will not only be as the retrovirus forerunner of HIV. In the 15-16 years since its discovery, practical public health and clinical therapeutic advances have come out of studies of its basic virology. Japan has eliminated or reduced breast feeding in HTLV-I endemic regions, and a blood test for HTLV-I is required in both the United States and Japan for blood donors to assure safety of the blood supply. These practices should substantially reduce HTLV-I infection. Rabbits can be infected with HTLV-I, and successful protection of them has been achieved with a vaccinia virus recombinant expressing the HTLV env gene. A successful vaccine against HTLV in humans would provide needed encouragement for the development of a vaccine against HIV. Perhaps more importantly, HTLV-I has also provided an experimental system to study the cause and pathogenesis of a human leukaemia and of chronic neurological disease with defined genetic tools. Although it has a far smaller clinical impact than HIV, in its more subtle role in human disease, HTLV-I may ultimately provide us with more fundamental information on the origin and pathogenesis of the more enigmatic chronic human diseases.

\section{HIV replication and selected aspects of pathogenesis}

No matter the current favourite view or the multiple models of HIV pathogenesis, virtually all depend on HIV replication. Consequently, the fine details of each of the steps involved in HIV replication merit intense study. HIV replication involves the usual retrovirus stages of cell surface binding, fusion of the virion and host cell lipid bilayers, reverse transcription, DNA integration, long terminal repeat (LTR)-regulated proviral expression, synthesis and proteolytic processing of structural proteins, assembly of viral RNA and proteins at the cell membrane, packaging, virion maturation and release. Special strategies employed by the HTLVs are also used by HIV. The HIV tat and rev genes encode nuclear proteins that have functional similarity to the regulatory genes of HTLVs, allowing production of HIV to occur in bursts. Tat and Rev bind to specific sites of HIV, and cellular factors are involved in this process. The relative conservation of their sequences, their need by HIV for significant levels of replication, and the fact that they offer more than one target - themselves or their target sequences - has 
made them prime candidates for anti-HIV therapy and perhaps the most intensely studied HIV genes and gene products ${ }^{33,34}$.

\section{Other HIV strategies}

HIV and other lentiretroviruses have still more genes (including nef, $v p r, v p u$, and vif) and other anti-HIV strategies relate to products of these and the structural genes ${ }^{33,34}$. For example, we first learned from HIV that special viral proteins may be released by acutely infected cells. As extracellular proteins they may potentiate HIV replication of nearby infected cells, facilitate infection of other cells or interfere with some immune functions. For example, Tat, Vpr and Nef may all be released and affect nearby cells. Tat promotes proliferation of some cells ${ }^{35}$ and expression of HIV in some latently infected cells, and it was recently shown to induce apoptosis of other $\mathrm{T}$ cells $^{36}$. Vpr has been reported to interfere with progression of the cell cycle by promoting a G2 arrest, perhaps thereby allowing more provirus expression and virus formation before the cell divides ${ }^{37}$. Vpr also appears to be necessary for HIV to infect macrophages. Along with the matrix antigen, p17, Vpr forms the preintegration DNA complex and aids its transport to the nucleus $^{38,39}$. In dividing cells, nuclear membrane disruption occurs, so the viral DNA can reach and interact with the chromosome. Non-dividing cells, like macrophages, retain nuclear membrane integrity. $\mathrm{Vpr}$ may be essential to macrophage infection by facilitating provirus entry into the nucleus in the absence of nuclear membrane breakdown ${ }^{39}$. Vpr is an excellent example of a viral gene product originally believed to be accessory, but which we have recently learned may have multiple intra- and extracellular functions aiding replication of HIV, and therefore is another target for our development of drugs that interfere with HIV replication ${ }^{40}$. Nef is another example. By binding to some cellular proteins Nef may contribute to T-cell activation or inhibition of activation, thereby influencing both HIV replication and Tcell survival ${ }^{41,42}$. If Nef can do the same as an extracellular protein, modulating uninfected cells, it would obviously markedly facilitate HIV expansion. These so-called auxiliary proteins are not the only HIV proteins released into the extracellular space. Gp120 is easily shed from virions and has been shown capable of binding CD4 of uninfected T cells impairing their function and survival, while downregulating CD4 of the infected cell. Downregulation of $\mathrm{CD}^{+}$infected cells also occurs from another 'auxiliary' protein, Vpu (ref 43). Vpu dissociates intracellular interactions of CD4 and the HIV envelope (gp160) so that the gp160 reaches the cell surface for viral formation while facilitating CD4 degradation. Downregulation of CD4 may prevent superinfection and thus rapid cell death before sufficient HIV replication.

\section{HIV infection}

Results over the past few years indicate far more HIV-1 replication than we first supposed. Nonetheless, there is no doubt that a state of viral 'latency' exists for many infected cells due to defective proviruses and in some instances probably to the unavailability of needed cellular transcription factors. Soluble factors such as some cytokines may also contribute. It is important to remember that latently infected cells not making virus may still make and release viral proteins, which contribute to pathogenesis.

Several different pathologic effects of HIV-1 infection appear constant: (1) eventual immune destruction associated with both immune deficiency (and associated opportunistic infections) and chronic immune activation; (2) neurological damage; and (3) hyperproliferation of some cell types, which may lead to neoplastic transformation. The differences among individuals in the extent of these consequences and the time it takes for them to occur depend on the HIV-1 variant, the age and very likely the genetics of the individual, and the individual's microbial environment. It is possible that some specific cofactors also contribute, but other than by increasing HIV replication (as some herpes viruses do), there is no evidence that any one is critical. It has become increasingly appreciated that it is the amount of HIV more than any other factor that determines the clinical outcome. Ironically, science has come full circle to the 1984 view that a direct effect of HIV infection is the obvious central aspect of HIV pathogenesis.

\section{HIV pathogenesis}

There is now abundant information on the mechanisms that may be involved in immune impairment and notably the fall in $\mathrm{CD}^{+} \mathrm{T}$ cells. The problem is to ascertain which of the many described in vitro mechanisms are important in vivo and which in vivo result is more important than another. HIV can kill activated $\mathrm{CD}^{+} \mathrm{T}$ cells. This effect may be mediated by perturbances in cell membrane integrity or by gp 120 interaction with CD4 or both. HIV-1 particles that are defective because of improper processing of core proteins are nonetheless able to enter some cells, and have been reported able to kill $\mathrm{T}$ cells (K. Van der Helm, pers. commun.). Also, isolated HIV proteins can affect Tcell survival or proliferation. For example, there are multiple mechanisms by which gp120 may cause loss or dysfunction of uninfected $\mathrm{CD}^{+} T$ cells, such as cross-linking of CD4, triggering apoptotic death after immune activation or CTL attack of uninfected $\mathrm{CD}^{+} \mathrm{T}$ cells binding gp120, which may have been shed from some virions. Some clinical results favour an interpretation that viral proteins alone may promote progression to AIDS. For instance, there is consensus that progression correlates with the plasma levels of HIV-1 RNA (reflecting viraemia), and there is evidence that a still greater rate of disease may occur in individuals with increasing levels of p24 while viral genomic RNA remains in check (J. Goudsmit, pers. commun.). This suggests that defective virions such as RNA-free particles and/or viral proteins expressed in the absence of particle formation contribute to AIDS pathogenesis. Molecular mimicry may 
also contribute either by provoking a harmful immune response, such as induction of antibodies to $\mathrm{T}$ cells, or by a competitive effect; for example (as recently described by J.F. Zagury and D. Zagury and their co-workers), the homology between regions of Env and the CD4 molecule may lead to inhibition of function of CD4 (ref. 44). Apoptotic death of a variety of uninfected 'bystander cells' located near HIV-infected $\mathrm{CD}^{+} \mathrm{T}$ cells, has been demonstrated in vivo by T.H. Finkel and her colleagues ${ }^{45}$, perhaps because of CTLs activated by the presence of nearby infected cells as recently suggested ${ }^{+6}$. Indeed, HIV-1 immune pathogenesis is associated with a state of chronic activation, but analogous to the difficulties related to clinical manipulation of pleiotropic cytokines, there is more than one side to consider. For example, chronic activation may lead to death of needed cells and to spread of HIV (both bad) but also to the development of cells with the means to target HIV and to guard against opportunistic infections (both good). Should we promote or inhibit activation?

There is also much confusion about the role of the thymus gland in the development of AIDS. We can assume that the thymus is damaged after HIV infection. This has led to plans for allogeneic thymus transplants for treating late-stage AIDS. Yet, we have little information on the presence, structure, function, and need of the thymus in normal human adults. Here there is a clear need for fundamental research: much more information on the biology and function of the thymus of the normal adult is required, although clinical trials may provide some of the answers.

Infected macrophages and related antigen-presenting cells do not usually die as a result of HIV infection, but may be functionally altered. However, after the early period of lymph node enlargement, due chiefly to hyperplasia of the B cells, gradual destruction of lymph nodes occurs and eventually includes degeneration of a major antigen presenting cell, the follicular dendritic cell (FDC) even though most FDC are not infected. FDCs trap virus in immune complexes as they do antigens in other infections. Probably, HIV is 'fed' to the trafficking CD4 $4^{+} \mathrm{T}$ cells, that are designed to be stimulated by this contact with antigen, but in this case the $\mathrm{T}$ cells become infected. After a long latency period, the FDC virus trapping ends and is associ-

Typical cytopathic effect observed in CD4 $4^{+} \mathrm{T}$-cell cultures infected by HIV. The picture represents different events observed in vitro after HIV infection of $\mathrm{CD}^{+} \mathrm{T}$ cells (in this case SUPT- 1 cells were infected with HIV- $2_{\text {SBL/SY }}$ ). $a$, Forming syncytium; $\quad b$, further recruitment of cells by the forming syncytium; and $c$, syncytium can become very large before lysis. (Figure courtesy of G. Franchini, Labouratory of Tumor Cell Biology, National Cancer Institute.) ated with an unexplained loss of the FDC and then the breakup of the lymph node architecture, correlating with viral spread from the compartmentalized lymph nodes and heralding the final stages of HIV infection ${ }^{20,21}$. There is extensive literature on the more cytopathic syncytia-inducing HIV variants (T tropic) and the less cytopathic non-syncytia-inducing HIV variants (macrophage tropic), and conflicting results about their overall contribution to pathogenesis. However, the meaning and clinical relevance of these assays is questionable, and if better therapy of infected patients is our objective, it hardly matters which view is correct since the clinical outcome appears to correlate only with the total amount of virus.

\section{HIV and cytokine production}

Alterations in production of some cytokines have been known almost from the earliest research on AIDS. For example, underproduction of IL-2 has led some to the therapeutic use of IL-2, whereas others have promoted clinical testing of IL-12, which promotes T-cell growth and development of CTLs, and restores $\mathrm{T}$-cell proliferation in vitro. However, cytokines have many faces and manipulation of them can result in multiple effects, the dominant one often not easy to predict. Nowhere is this better illustrated than with the interferons. Both IFN- $\alpha$ and IFN- $\gamma$ levels are often increased in HIV-1-infected people ${ }^{t}$. Indeed, IFN- $\alpha$ is sometimes used to treat HIV infection because of its anti-HIV and anti-Kaposi's sarcoma (KS) effects. However, its capacity to also block T-cell proliferation and even to induce apoptosis is the other side of the face, and it is quite possible that IFN- $\alpha$ is, in fact, a major factor in HIV immune pathogenesis as suggested by Zagury ${ }^{+4}$. Should we add more IFN- $\alpha$ or inhibit it? Which is better? Similar dilemmas arise with IFN- $\gamma$. Their levels are also increased but their efficacy may be impaired by anti-IFN antibody production ${ }^{47}$. Some trials of IFN- $\gamma$ were initiated in the hopes it would augment immunity but were associated with promotion of Kaposi's sarcoma, a phenomenon predicted by some in vitro studies (see below).

\section{HIV and AIDS}

Recent reports suggest much more HIV-cell interaction with greater cell and viral turnover and more profound drop in virus titre with some therapy than previously known ${ }^{18,19}$. These studies were sometimes heralded as 'good news' because of the precipitous fall in HIV following therapy and the apparently extensive $\mathrm{T}$-cell proliferative capacity in replacing lost cells. In reality the evidence for the $\mathrm{T}$-cell proliferative response is inconclusive because of the lack of information on normal human T-cell turnover and compartmentalization, and the escape of virus from those drugs and its apparent quick spread and associated $\mathrm{CD} 4^{+} \mathrm{T}$-cell decline serve to remind us what has been known since 1984: HIV-1 causes AIDS and by one way or another leads to $\mathrm{CD} 4^{+} \mathrm{T}$-cell loss.

A major focus in recent years has been on people with HIV-1 infection who do not show any significant signs of AIDS or of immune impairment despite long-term infection ('long-term non-progressors'). Several groups have studied most of their immune parameters. The results are clear: these normal-appearing people have normal or near normal parameters of immune function and lymph node architecture. No common cofactor (favouring health) or absent cofactor (favouring progression) have been identified, yet these individuals handle HIV much better than most. The reason(s) must lie in the strain of HIV-1 that infected them, the dose of the virus at the 
time of infection, or some aspect of their genetics. Regarding the latter, most discussions have logically focused on the genetics controlling the fundamental way they 'see' or respond to HIV, that is, the type of immune response. However, there are many other possibilities. As do all viruses, HIV depends on numerous host factors for its replication, and as any component of the cell does, these host factors vary from individual to individual and could influence the tropism of HIV and its rate and level of replication.

\section{HIV-1 and neoplasias}

Two unusual concepts in virology have emerged, which if not original with HIV-1 and its role in neoplasias, have been more emphasized: the role of extracellular viral proteins in pathogenesis, and the indirect role of a virus in tumorigenesis.

Kaposi's sarcoma. Kaposi's sarcoma is rare in the absence of HIV infection and occurs in fewer than 1 per 100,000 per year, but most frequently in older Ashkenazi Jews, Mediterraneans, equatorial Africans and recipients of organ transplants. Associated with HIV-1 infection, the incidence is increased three- to fourfold in blood transfusion recipients, several-fold in intravenous drug addicts, and much more (thousands-fold) in homosexual males. Strangely, males dominate all forms of $\mathrm{KS}$. Although many researchers have reported finding microbial links (varying from viruses to mycoplasma), the recent discovery of nucleotide sequences of a new human herpesvirus, HKSV HHV -8 associated with KS is the first that has been reproducibly established as a link to KS and might be an essential aetiological agent $t^{48}$. This virus is composed of some sequences related to Epstein-Barr virus (EBV), but more closely to herpes saimiri, a virus of squirrel monkeys. However, the key enigmatic features remain, such as the unexplained male preponderance, apparent multifocal appearance, composition (early on as a mixture of cell types with a predominance of vascular spindle-shaped cells and accompanying extensive angiogenesis), the question of whether it is a malignancy or a hyperplasia, which cell is the true tumour cell, and the mechanistic role of the viruses HIV-1 and HHV-8. Nonetheless, studies on the pathogenesis of KS and the possible role of HIV-1 have substantially progressed in the past several years following development of cell culture systems and murine in vivo models ${ }^{49} 52$. These results indicate that the majority of cultured cells are hyperplastic, not neoplastic.

However, we recently isolated malignant cells (called KS Y-1) from a late-stage HIV-1-associated KS for the first time ${ }^{52}$. Another earlier example was found in one case of classical $\mathrm{KS}^{53}$. These cells provide the first evidence for malignant cells in KS. We find the HIV-1 sequences present only in a minority of cells of a KS tumour and present neither in the hyperplastic spindle cells nor the neoplastic cells. Consequently, any role of HIV-1 in $\mathrm{KS}$ appears to be indirect. Our results suggest that it is at least twofold: augmenting inflammatory cytokine production, perhaps especially IFN- $\gamma$, and the production and release of the HIV-1 Tat protein, which promotes endothelial cell growth, adhesion, and invasiveness synergistic with bFGF.

The role of HHV-8 is yet to be defined. It has generally been assumed that it acts as a true tumour virus, transforming its target cells. However, our evidence suggests that like HIV-1, HHV-8 is not in most KS tumour cells. Moreover, we have found HHV-8 sequences in peripheral blood lymphocytes (PBLs) of several normal individuals. That result, coupled with the fact that KS associated with kidney transplantation (from many different donor sources) is also HHV-8 positive, suggests that HHV-8 may be much more widespread than suggested, and that the finding of this virus may not explain the very reason it was sought: namely, the greater incidence of all forms of $\mathrm{KS}$ in men and its extraordinary incidence in HIV-1-infected homosexual men. If a causative role of HHV-8 in $\mathrm{KS}$ can be ascribed, it may be in augmenting release of some cytokines, like the ones described above, and therefore as for HIV-1 an indirect role in KS but with linkage to all KS forms.

Overall, our results indicate that KS evolves from hyperplasia to malignancy. The malignant clones could develop from chance genetic alterations from the hyperplastic KS spindle cell. Recently, my colleagues and I made a fortuitous discovery. We found that both the hyperplastic KS spindle cells and the neoplastic cells undergo apoptosis when treated with a hormone of pregnancy, the beta chain of human chorionic gonadotropin. Consequently, human chorionic gonadotropin is now in clinical trials for KS.

B-cell lymphoma. Unlike KS, immune deficiency is clearly linked with development of B-cell lymphomas in HIV-1- and HIV-2-infected persons and in SIV-infected macaques. As in KS, HIV sequences are not found in the tumour cells. The mechanism may involve chronic polyclonal B-cell stimulation mediated by gp120 and gp41 and high levels of the B-cell growth-promoting cytokines, IL-6, and IL-4 in HIV-infected people. Along with other cytokines (such as IL-10, which decreases apoptosis of B cells), these factors may increase the probability of B-cell neoplastic transformation. EBV sequences are present only in a fraction of these tumours. Whether there is a role for another immortalizing virus is unknown. Our hypothesis that there might be one led to our search for and discovery of a new human herpesvirus, HHV-6 (ref. 54), but ironically HHV-6 turned out not to be a B-cell growth-promoting virus, but one that infects and impairs $\mathrm{T}$ cells.

\section{AIDS research: Is it at a crossroad?}

Effective therapy of HIV-infected people has not yet been successful, and no vaccine with high-level success can be promised for the immediate future. The great resources for AIDS research and a frustrated vocal activist group combined to recently generate discussions and debates in and outside of the scientific community that have questioned the direction, priorities and the effectiveness of the scientific administrative leadership of AIDS research. The failures of therapy and vaccines stand in contrast to the rapid advances made in the early period of AIDS research (1983-85), a period called the years of intense discovery by J. Mann. As compared with these results in only a few year's time, major results of subsequent years (1986-1995) may, in comparison appear meager, particularly given the large number of scientists involved. However, our appreciation of the real progress in this period should not be clouded. We have learnt much about the role of the HIV 'auxiliary' genes, developed new animal model systems, gained a greater appreciation for clinical experiments and of individual variation in resistance to disease and come to understand the importance of immune parameters that control HIV replication. Also, recent years have witnessed an explosion of information on various cytokines, the crystallization of some HIV-1 proteins, an appreciation of the value of combination therapy against HIV, fostered a much better understanding of KS and of overall immune pathogene- 
sis. We have seen the development of several new anti-HIV agents and anti-KS agents, realize that many approaches will not work for an HIV vaccine and now know that there is much more HIV in an infected person than we realized in the early years. Nonetheless, the frustration remains, because the major scientific advances awaited are nothing less than a complete cure of infected people and a preventive vaccine. The response has been either a call for more basic research or for more careful planning and more targeting of some specific issues. The 'argument' for more basic research is not a real issue. No one doubts its need. The questions must be more specific: What will the definition of basic research be and who will decide what is included as 'basic'? What will be reduced in the budget to accommodate it and who will make those decisions? In my experience major advances in therapy or public health are preceded not only by active laboratory research but also diverse clinical trials based on different ideas from different investigators. Consequently, any reduction in such trials must carry the greatest concern.

Therapy and vaccines in the future. All of the known mechanisms by which HIV impairs the immune system depend on HIV replication; consequently, the development of many antiHIV agents and their clinical testing alone and in various combinations and as early after infection as possible are the obvious priorities. The recent findings that some anti-HIV agents produce a profound decline in virus levels within a few weeks of treatment will make effective drug screening in vivo a reality, but there must be more rigorous attempts to avoid drug resistance. Targeting conserved and critical viral amino acid or nucleotide sequences is one way. As we recently proposed, targeting cellular factors (like ribonucleotide reductase with hydroxyurea) $)^{55}$, which the virus may need more than the cell and which is not subject to the variation of HIV, is another.

Gene therapy offers hope. There is no doubt that cells transduced with HIV inhibitory genes can protect those same cells from infection in vitro. Plans for ex vivo transduction of such genes into $T$ cells of patients and reinfusing these cells is ongoing. There are uncertainties - such as obtaining a sufficient number of stem cells successfully transduced. A major advance would be the development of vectors that could deliver the gene to stem cells by direct injection into the patient. Knowing whether the inhibitory gene will be stable and not toxic is also important. If these problems are solved there still remains the problem of infection of the brain. Clinical trials should answer some of these questions. Manipulation of cytokine levels will also be a focus of therapy in the coming years, as well as many different approaches.

Research for an HIV preventive vaccine presents some special difficulties: the limits in the utility of the current animal models, the failure so far to define immune parameters of protection for humans, the great variability of HIV, the relatively short-lived immune response to HIV immunogens, the possibility of inducing enhancing antibodies that facilitate rather than inhibit infection, and the apparent declining interest by the pharmaceutical and vaccine companies. All of these argue strongly to hold back from efficacy field testing, as indeed has been the decision in the United States. Yet, many of the same arguments can be used to argue for such field trials. For example, without human testing, we may never know the immune parameters to seek. It is not hard then to sympathize with those companies and agencies (such as the World Health
Organization) that are going forward in areas of extreme high risk. The right vaccine preparation, of course, is not known. From the available information it is difficult to believe that purified HIV proteins will be effective. The desperation in some countries has led some to propose using 'live' infectious but 'crippled' HIV by deleting the nef gene or multiple auxiliary genes. This was based on success with nef-deleted SIV in monkeys. To go forward in a vaccine trial in normal humans with such attenuated but still infectious HIV is a course I have been strongly against. The time period of observation of the monkeys is far too short, and our experiences from chickens to humans have taught us that any infectious retrovirus, even one poorly replicating, may ultimately cause disease. Finally, R. Ruprecht and her colleagues recently showed that such 'immunogens' do indeed cause AIDS when used in infant monkeys ${ }^{56}$. I favour the intermediate course - neither purified proteins delivered as such nor live attenuated HIV, but other attenuated viral vectors like attenuated poxvirus or adenovirus as recombinants with HIV genes. Results with them have been encouraging. For instance, in collaboration with E. Paoletti and J. Tartaglia we have shown that this approach can be successful in protecting animais against infection with HTLV-I and HIV-2. However, for HIV-1 we need more fundamental research on ways to broaden, increase and sustain the immune response.

It is my impression that some pharmaceutical companies are slowly moving away from major long-term commitments to development of an HIV vaccine. The combination of a lack of sufficient success in vaccine development, the threat of lawsuits and criticisms from activists serves to foster their disengagement. The lack of sufficient incentives makes vaccine development a research area that begs for long-term targeted research efforts, financial commitment from the nations of the world and effective leadership. It is often said that competition is divisive and cooperation noble even when the former catalyzes success and the latter, sometimes nothing more than niceties, but in the arena of development of an HIV effective vaccine cooperation is now mandatory.

Laboratory of Tumor Cell Biology, Building 37, Room 6 A09

National Cancer Institute,

National Institutes of Health

37 Convent Drive

Bethesda, Maryland 20892-4255

\section{Acknowledgements}

It is a pleasure to acknowledge and to thank my colleagues, Flossie WongStaal, Mariorie Robert-Guroff, Genoveffa Franchini, William Blattner and Marvin Reitz for reading this manuscript in advance and making helpful comments. I also am grateful to Cecil Fox for his helpful discussions. Finally, I thank Anna Mazzuca for her editorial assistance. For editorial reasons, it was not possible to cite many other important contributions.

1. Temin, H.M. Origin and general nature of retrovirus. The Retroviridae, vol. 1 (ed. Levy, J.A.) (Plenum, New York, 1992).

2. Coffin, J.M. Structure and classification of retroviruses. The Retroviridae, vol. 1 (ed. Levy, J.A.) (Plenum, New York, 1992).

3. Gallo, R.C. The story of retroviruses and cancer: from poultry to people. in Virus Hunting 59-81 (Basic Books, New York, 1991).

4. Lower, R. et al. Identification of human endogenous retroviruses with complex mRNA expression and particle formation. Proc. natn. Acad. Sci. U.S.A. 90, 4480-4484 (1983).

5. Barré-Sinoussi, F. et al. Isolation of a T-lymphotropic retrovirus from a patient at risk for acquired immune deficiency syndrome (AIDS). Science 220, 868-871 (1993).

6. Popovic, M., Sarngadharan, M.G., Read, E. \& Gallo, R.C. Detection, isolation, and continuous production of cytopathic retroviruses (HTLV-III) from pa- 
tients with AIDS and pre-AIDS. Science 224, 497-500 (1984).

7. Gallo, R.C. et al. Frequent detection and isolation of cytopathic retroviruses (HTLV-III) from patients with AIDS and at risk for AIDS. Science 224, 500-503 (1984).

8. Sarngadharan, M.G., Popovic, M., Bruch, L., Schupbach, J. \& Gallo, R.C. Antibodies reactive with human T-lymphotropic retroviruses (HTLV-III) in the serum of patients with AIDS. Science 224, 506-508 (1984).

9. Gallo, R.C. The first human retrovirus. Sci. Am. 255 (Dec.), 88-98 (1986).

10. Poiesz, B.J. et al. Detection and isolation of type $C$ retrovirus particles from fresh and cultured lymphocytes of a patient with cutaneous $\mathrm{T}$-cell lymphoma. Proc. natn. Acad. Sci. U.S.A. 77, 7415-7419 (1980).

11. Miyoshi, I. et al. Type $C$ virus particles in a cord T-cell line derived by co-cultivating normal human cord leukocytes and human leukaemic T cells. Nature 294, 770-771 (1981).

12. Gallo, R.C. Discovery of a cancer virus: The first human retrovirus. in Virus Hunting 99-115 (Basic Books, New York, 1991).

13. Franchini, G. Molecular mechanisms of HTLV-I infection. Blood (in the press).

14. Kalyanaraman, V.S. et al. A new subtype of human T-cell leukemia virus (HTLV-II) associated with a T-cell variant of hairy cell leukemia. Science 218 , 571.573 (1982).

15. Lairmore, M.D. et al. Isolation of human T-cell lymphotropic virus type 2 from Guaymi Indians in Panama. Proc. natn. Acad. Sci. U.S.A. 87, 8840-8844 (1990).

16. Robert-Guroff, M. et al. Prevalence of antibodies to HTLV-I, -II, and-III in intravenous drug abusers from an AIDS endemic region. JAMA 255, 3133-3137 (1986).

17. Zagury, D. et al. Long-term cultures of HTLV-III-infected T cells: A model of cytopathology of T-cell depletion in AIDS. Science 231, 850-853 (1986).

18. Wei, X. et al. Viral dynamics in human immunodeficiency virus type 1 infection. Nature 373, 117-122 (1995).

19. Ho, D.D. et al. Rapid turnover of plasma virions and CD4 lymphocytes in HIV-1 infection. Nature 373, 123-126 (1995).

20. Pantaleo, G., Graziosi, C. \& Fauci, A.S. The role of lymphoid organs in the pathogenesis of HIV infection. Semin. Immun. 5, 157-163 (1993).

21. Embretson, J. et al. Analysis of human immunodeficiency virus-infected tissues by amplification and in situ hybridization reveals latent and permissive infections at single-cell resolution. Proc. natn. Acad. Sci. U.S.A. 90, 357-361 (1993).

22. Catovsky, D. et al. Adult T-cell lymphoma-leukaemia in Blacks from the West Indies. Lancet 1, 639-643 (1982).

23. Robert-Guroff, M. et al. Natural antibodies to human retrovirus HTLV in a cluster of Japanese patients with adult T cell leukemia. Science 215, 975-978 (1982).

24. Bartholomew, C. et al. HTLV-I and tropical spastic paraparesis [letter]. Lancet 2, 99-100 (1986).

25. Grassmann, R. et al. Transformation to continuous growth of primary human T lymphocytes by human T-cell leukemia virus type I X-region genes transduced by a Herpesvirus saimiri vector. Proc. natn. Acad. Sci. U.S.A. 86, 3351-3355 (1989).

26. Tanaka, A. et al. Oncogenic transformation by the tax gene of human T-cel leukemia virus type I in vitro. Proc. natn. Acad. Sci. U.S.A. 87, 1071-1075 (1990).

27. Saggioro, D. et al. Tax protein of human T-lymphotropic virus type I triggers DNA damage. Leuk. Lymphoma 12, 281-286 (1994).

28. Gessain, A. et al. Antibodies to human T-lymphotropic virus type-I in patients with tropical spastic paraparesis. Lancet 2, 407-410 (1985).

29. Osame, M. et al. HTLV-I associated myelopathy, a new clinical entity [letter]. Lancet 1, 1031-1032 (1986).

30. Waldmann, T.A. Anti-IL-2 receptor monoclonal antibody (anti-Tac) treatment of T-cell lymphoma. Important Adv. Oncol. 131-141 (1994).

31. Gill, P.S. et al. Treatment of adult T-cell leukemia-lymphoma with a combination of interferon alfa and zidovudine. New Engl. J. Med. 332, 1744-1748 (1995).

32. Hermine, O. et al. Brief report: treatment of adult T-cell leukemia-lymphoma with zidovudine and interferon alfa. New Engl. J. Med. 332, 1749-1751 (1995)

33. Haseltine, W.A. \& Wong-Staal, F. The molecular biology of the AIDS virus. Sci. Am. 259 (Oct.), 52-62 (1988)

34. Cullen, B.R. \& Greene, W.C. Functions of the auxiliary gene products of the human immunodeficiency virus type 1. Virology 178, 1-5 (1990).

35. Ensoli, B., Barillari, G., Salahuddin, S.Z., Gallo, R.C. \& Wong-Staal, F. Tat protein of HIV-1 stimulates growth of cells derived from Kaposi's sarcoma lesions of AIDS patients. Nature 345, 84-86 (1990).

36. Li, C.J., Friedman, D.J., Wang, C., Metelev, V. \& Pardee, A.B. Induction of apoptosis in uninfected lymphocytes by HIV-1 Tat protein. Science 268, 429--431 (1995).

37. Rogel, M.E., Wu, LI. \& Emerman, M. The human immunodeficiency virus type I vpr gene prevents cell proliferation during chronic infection. I. Virol. $69,882-888$ (1995).

38. Bukrinsky, M.I. et al. A nuclear localization signal within HIV-1 matrix protein that governs infection of non-dividing cells. Nature 365, 666-669 (1993).

39. Bukrinsky, M.I. et al. Active nuclear import of human immunodeficiency virus type 1 preintegration complexes. Proc. natn. Acad. Sci. U.S.A. 89, 6580-6584 (1992).

40. Balotta, C., Lusso, P., Crowley, R., Gallo, R.C. \& Franchini, G. Antisense phosphorothioate oligodeoxynucleotides targeted to the vpr gene inhibit human immunodeficiency virus type 1 replication in primary human macrophages. J. Virol. 67, 4409-4414 (1993).

41. Saksela, K., Cheng, G. \& Baltimore, D. Proline-rich (PxxP) motifs in HIV-1 Nef bind to SH3 domains of a subset of Src kinases and are required for the enhanced growth of Nef+ viruses but not for down-regulation of CD4. EMBO I. 14, 484-491 (1995).

42. Baur, A.S. et al. HIV-1 Nef leads to inhibition or activation of $\mathrm{T}$ cells depending on its intracellular localization. Immunity 1, 373-384 (1994).

43. Strebel, K., Klimkait, T. \& Martin, M.A. A novel gene of HIV-1, vpu, and its 16-kilodalton product. Science 241, 1221-1223 (1988).

44. Zagury, J.F. et al. Identification of CD4 and major histocompatibility complex functional peptide sites and their homology with oligopeptides from human immunodeficiency virus type 1 glycoprotein gp120: Role in AIDS pathogenesis. Proc. natn. Acad. Sci. U.S.A. 90, 7573-7577 (1993).

45. Finkel, T.H. et al. Apoptosis occurs predominantly in bystander cells and not in productively infected cells of HIV- and SIV-infected lymph nodes. Nature Med. 1, 129-134 (1995).

46. Pantaleo, G. \& Fauci, A.S. Apoptosis in HIV infection. Nature Med. 1, 118-120 (1995).

47. Turano, A. et al. Natural human antibodies to interferon-interfere with the immunomodulating activity of the lymphokine. Proc. natr. Acad. Sci. U.S.A. $89,4447-4451$ (1992)

48. Chang, Y. et al. Identification of herpesvirus-like DNA sequences in AIDS-associated Kaposi's sarcoma. Science 266, 1865-1869 (1994).

49. Nakamura, S. et al. Kaposi's sarcoma cells: Long-term culture with growth factor from retrovirus-infected CD4* T cells. Science 242, 426-430 (1988).

50. Ensoli, B. et al. Synergy between basic fibroblast growth factor and HIV-1 Tat protein in induction of Kaposi's sarcoma. Nature 371, 674-680 (1994).

51. Ensoli, B. \& Gallo, R.C. AIDS-associated Kaposi's sarcoma: A new perspective of its pathogenesis and treatment. Proc. Assoc. Am. Physicians 107, 8-18 (1995).

52. Lunardi-Iskandar, Y. et al. Tumorigenesis and metastasis of neoplastic Kaposi's sarcoma cell line in immunodeficient mice blocked by a human pregnancy hormone. Nature 375, 64-68 (1995).

53. Siegal, B. et al. Kaposi sarcoma in immunosuppression. Possibly the result of a dual viral infection. Cancer 65, 492-498 (1990).

54. Salahuddin, S.Z. et al. Isolation of a new virus, HBLV, in patients with lymphoproliferative disorders. Science 234, 596-601 (1986).

55. Lori, F. et al. Hydroxyurea as an inhibitor of human immunodeficiency virus-type 1 replication. Science 266, 801-805 (1994).

56. Baba, T.W. et al. Pathogenicity of live, attenuated SIV after mucosal infection of neonatal macaques. Science 267, 1820-1825 (1995). 